\title{
Subwavelength atom localization via coherent population trapping
}

\author{
G S Agarwal ${ }^{1,3}$ and K T Kapale , $^{2,4}$ \\ ${ }^{1}$ Department of Physics, Oklahoma State University, Stillwater, OK 74078, USA \\ 2 Jet Propulsion Laboratory, California Institute of Technology, Mail Stop 126-347, \\ 4800 Oak Grove Drive, Pasadena, CA 91109-8099, USA
}

Received 8 April 2006, in final form 30 June 2006

Published 14 August 2006

Online at stacks.iop.org/JPhysB/39/3437

\begin{abstract}
We present an atom localization scheme based on coherent population trapping. We consider atomic transitions in a Lambda configuration where the control field is a standing-wave field. The probe field and the control field produce coherence between the two ground states and prepare the atom in a pure state. We show that the population in one of the ground states has the same fringe pattern as produced by a Fabry-Perot interferometer and thus measurement of this population would localize the atom. Interestingly enough the role of the cavity finesse is played by the ratio of the intensities of the pump and probe. This is in fact the reason for obtaining extreme subwavelength localization.
\end{abstract}

\section{Introduction}

Precision position measurement of an atom has been of interest since the early days of quantum mechanics. An illustrative example is the Heisenberg microscope which allows the measurement of the position of an atom by observing scattering of light from it. Modern tools of quantum optics have made such thought experiments a reality. A variation of Heisenberg's microscope has been studied in [1]; it allows suboptical wavelength position measurements of moving atoms as they pass through the optical fields. Since then a variety of methods have been studied for subwavelength localization of an atom passing through standing-wave fields. Subwavelength atom localization has been shown to be possible through quantum interference [2], by measuring a quadrature phase of the light field in a cavity as the atom passes through it [3], through detection of spontaneously emitted photons [4, 5], by diffracting the atoms through a measurement-induced grating [6] or by Raman-induced resonance imaging [7]. Recently, phase control of subwavelength atom localization has also been shown giving rise to a variety of interesting controllable features in atom localization [8]. Similar techniques could also be used to perform the measurement of the atomic centre-of-mass wavefunction as

\footnotetext{
3 On leave from Physical Research Laboratory, Navrangpura, Ahmedabad-380 009, India.

4 Also at Hearne Institute of Theoretical Physics, Department of Physics and Astronomy, Louisiana State University, 202 Nicholson Hall, Tower Drive Baton Rouge, LA 70803, USA.
} 


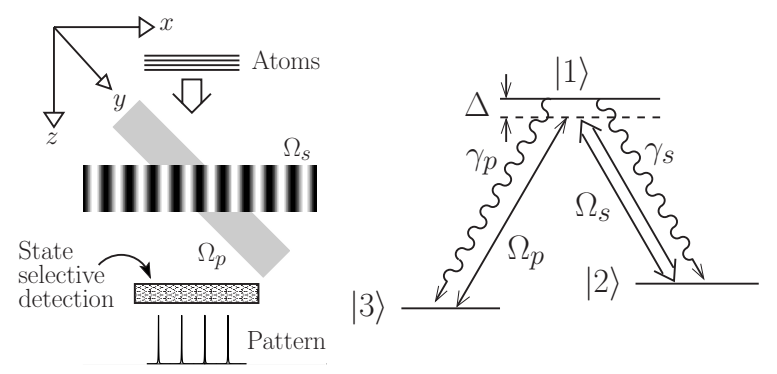

Figure 1. Application of a strong standing-wave field, on the $|1\rangle-|2\rangle$ transition, and a weak Probe field, on the $|1\rangle-|3\rangle$ transition, prepares the atom in a particular position-dependent superposition of the states $|2\rangle$ and $|3\rangle$ in steady state. Probing this superposition through selective measurement of the population of the state $|2\rangle$ causes subwavelength localization of the atom at the nodes of the standing-wave field.

proposed recently by one of us and collaborators [9]. Recently relative position localization through entanglement has also been shown to be possible [10].

The study of atom localization affords practical applications in the area of nanolithography at the Heisenberg limit $[11,12]$ along with its fundamental importance in the areas of atom optics [13] and laser cooling [14] and trapping of neutral atoms [15]. In this paper we propose a high-resolution localization scheme based on the phenomena of coherent population trapping (CPT) [16]. A standing-wave control field and a weak probe field cause the atom to evolve into a long-lived superposition of the two ground states. Measuring the position-dependent coherence of this trapping state or the population of one of the ground states localizes the atom. Extending the scheme to two dimensions, optical lattices with tighter than usual confinement at each lattice point can be obtained. Such a strongly confined lattice structure could be useful to study several predictions of the Bloch theory of solids and Mott transitions in much cleaner systems compared to the conventional solids.

The paper is organized as follows. First we discuss the scheme of localization through the phenomena of coherent population trapping. As the localization of the atom occurs through the detection of a population we provide a detection scheme that would lead to localization of the atom in a subwavelength regime as it passes through the standing-wave field. To ascertain the localization we present the momentum distribution of the localized atom and point out unique features that, if seen experimentally, would confirm the localization of the atom in the subwavelength domain. Next, we point out several applications of the scheme and the unique advantages it offers. Finally, we present our conclusions. In the appendix, we show that the system reaches a pure state at steady state even in the presence of recoil due to spontaneous emission.

\section{Localization scheme}

The level scheme for our model is depicted in figure 1 (cf [6]) along with a possible experimental geometry. The atom has $\Lambda$-type level configuration, such that it is coupled to two fields in two-photon resonance Raman configuration as shown in the figure. A possible atomic specie could be ${ }^{85} \mathrm{Rb}$ with the levels $|2\rangle$ and $|3\rangle$ drawn from the two hyperfine components of the ground state. The strong control field is the standing-wave field that could be a field confined in an optical cavity or a Fabry-Perot resonator or produced using two counter-propagating laser beams. The Rabi frequencies of the standing-wave field $\left[\Omega_{s}(x)=\Omega_{s} \sin (k x)\right.$, with the 
wavenumber $k=2 \pi / \lambda]$ and the weak probe field $\left(\Omega_{p}\right)$, could be of the order of tens of $\mathrm{MHz}$ and the laser power level could be few $\mathrm{mW} \mathrm{cm}^{-2}$ as in a typical CPT experiment [13]. The laser linewidths need to be smaller than the spontaneous decay width of the excited state ${ }^{5}$. The laser fields are along the $x-y$ plane as shown in figure 1 , such that the atom sees them at the same time as it travels along the $z$ direction.

The initial state of the atoms is $|3\rangle$ as they enter the fields in the transverse $z$ direction with the centre-of-mass distribution uniformly spread over a few standing-wave field wavelenghts. We assume that the velocity of the atoms in the $z$ direction can be treated classically and there is no significant variation of the $x$-velocity of the atoms as they interact with the fields; therefore, the kinetic energy of the atoms can be ignored within the Raman-Nath approximation (RNA) [17]. This poses a limitation on the extent of the localization possible as stricter confinement along the $x$ direction causes increasing spread in the $x$-momentum of the atom. However, RNA requires the recoil kinetic energy, $\hbar^{2} k_{a}^{2} / 2 m$, acquired by the atom of mass $m$ due to its $x$-momentum, $k_{a}$, to be significantly less than the interaction energy with the fields, $\hbar \Omega_{p}$ and $\hbar \Omega_{s}$, i.e.,

$$
\frac{\hbar^{2} k_{a}^{2}}{2 m} \ll \hbar \Omega_{p}, \hbar \Omega_{s}
$$

throughout the interaction region. This means that the interaction region should be sufficiently small so that the total acquired kinetic energy by the atom is small compared to its interaction energy with the optical fields. We show later that we remain within the limits of this regime of parameters throughout to achieve localization.

The interaction of the fields with the three-level atom, within the two-photon resonance condition, can be described through the interaction Hamiltonian

$$
\mathscr{H}=-\hbar\left(\Omega_{p}|3\rangle\left\langle 1\left|+\Omega_{s}(x)\right| 2\right\rangle\langle 1|\right) \mathrm{e}^{-\mathrm{i} \Delta t}+\text { h.c. }
$$

A quick observation shows that the state

$$
|\Psi\rangle=\left(\Omega_{p}|2\rangle-\Omega_{s}(x)|3\rangle\right) / \Omega,
$$

where $\Omega=\sqrt{\left|\Omega_{p}\right|^{2}+\left|\Omega_{s}(x)\right|^{2}}$, does not evolve dynamically as $\mathscr{H}|\Psi\rangle=0$. Thus, an atom initially prepared in the state $|3\rangle$ will end up in the state $|\Psi\rangle$ at steady state, if the two-photon resonance is maintained. As the population in the state $|\Psi\rangle$ cannot escape, it is termed as the trapping state and the phenomenon is called coherent population trapping. This state, being a superposition of ground states, does not decohere. There could be a physical loss of atoms during the transit time; however, this is not a limitation as these atoms would not be localized. It is, however, imperative to note that the time required to reach the CPT state in the steady state is several times the spontaneous decay time of the excited state $|1\rangle$. Thus, the interaction time has to be chosen such that it is sufficiently large compared to the lifetime of the excited state and sufficiently small compared to the time in which the energy extracted by the atom from the field is small enough that the Raman-Nath approximation remains valid. In this context, it can be noted that the recoil of the atoms due to spontaneous emission from excited state should be taken into account. We present the detailed treatment in the appendix and only state the result here that the pure state represented by the CPT state of equation (3) is still the steady state of the system.

Thus, ensuring the two-photon resonance, introduces coherence $\rho_{23}=-\Omega_{p} \Omega_{s}^{*}(x) / \Omega^{2}$ between the levels $|2\rangle$ and $|3\rangle$ that carries the spatial dependence of the standing-wave field. Also, as the CPT state $|\Psi\rangle$ is reached, the population of the state $|2\rangle$ is given by

$$
\rho_{22}(x)=1 /\left(1+\mathscr{R} \sin ^{2} k x\right),
$$

5 The laser linewidths must be smaller than the spontaneous decay width of the excited state, otherwise frequency locking of the control and probe lasers will become necessary. 

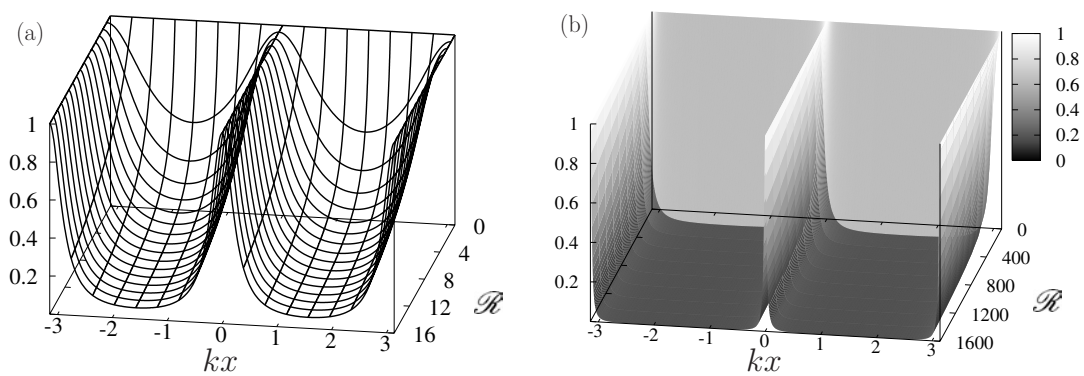

Figure 2. Emergence of subwavelength localization as the ratio $\mathscr{R}$ is increased. Plot of $\rho_{22}(x)$ versus $k x$ for various values of $\mathscr{R}$. The range of $k x,\{-\pi, \pi\}$, covers one wavelength of the standing-wave field. (a) For $\mathscr{R}=0, \rho_{22}$ is uniform at all spatial points $k x$. (b) Increasing the cavity field strength localization peaks emerge with decreasing peak width.

where $\mathscr{R}=\left|\Omega_{s}\right|^{2} /\left|\Omega_{p}\right|^{2}$. Therefore, so long as the steady state is reached within the interaction time (typically $100 \mathrm{~ns}$ ) governed by the $z$-velocity of the moving atoms, monitoring the population of the state $|2\rangle$ is a sufficient measure of the coherence of the CPT state. This interaction time is well below the limit of $<7 \mu$ s required to remain within the RNA for ${ }^{85} \mathrm{Rb}$ atoms undergoing optical transitions [6]. Our numerical study, considering both Gaussian and flat-top beams, shows that the spatial intensity profile is inconsequential if the trapping state is reached in the steady state within the interaction time. To add to the physical meaning of the above result, it could be noted that all the atomic density-matrix elements will have position dependence. Integrating the sum of populations in the three atomic levels over the width of the atomic beam would give the number of atoms passing the interaction region per unit time.

We plot the population $\rho_{22}$ versus $k x$ in figure 2 for various values of the ratio $\mathscr{R}$.

The population shows peaks at the nodes of the standing-wave field. The FWHM of the peaks is given by $k \Delta x=2 / \sqrt{\mathscr{R}}$. Thus, for small values of the ratio of the Rabi frequencies the peaks are not well defined. In fact, for $\mathscr{R}=0, \rho_{22}$ has no spatial dependence. The small $\mathscr{R}$ behaviour is shown in part $(a)$ of the figure where the emergence of the localization peaks is clearly seen. For large $\mathscr{R}$, the peaks become quite sharp, leading to subwavelength localization.

It can be noted that (4) has the same structure as the transmission function of the FabryPerot cavity [18], with the ratio $\mathscr{R}$ of the effective field intensities playing the role of the cavity finesse. Interestingly, in the present model the finesse can be controlled by varying the relative intensities of the control and probe fields, leading to much sharper features in $\rho_{22}$ (see figure 2).

It is imperative to point out the meaning of the result obtained above. The state vector of the atom, including its centre-of-mass degree of freedom and the CPT internal state, can be written as

$$
\left|\Psi_{\mathrm{COM}}\right\rangle=\int f(x)|x\rangle \frac{1}{\Omega}\left(\Omega_{p}|2\rangle-\Omega_{s}(x)|3\rangle\right) \mathrm{d} x,
$$

where $f(x)$ is the centre-of-mass wavefunction. Thus, observance of a peak in $\rho_{22}$ amounts to a collapse of the internal state of the atom to $|2\rangle$ at one of the positions corresponding to the nodes of the standing-wave field. This leads to the collapse of the centre-of-mass wavefunction at one of these positions leading to localization of the atom. With sharper features in $\rho_{22}(x)$ one obtains deeper localization of the atom within a subwavelength region of the optical field. Our treatment assumes that $f(x)$ is uniform over the standing-wave field, which remains 


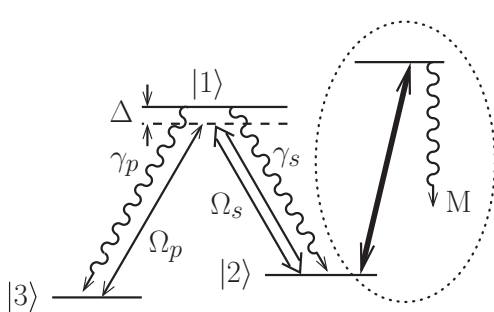

Figure 3. Technique to monitor the atom localization. The CPT state of the atom could be probed by monitoring the population of the state $|2\rangle$, i.e., $\rho_{22}(x)$. Efficient measurement of $\rho_{22}(x)$ can be accomplished via the fluorescence shelving technique as depicted in the figure. Applying a strong shelving field and monitoring the resulting fluorescence leads to localization of the atom at the nodes of the standing-wave field.

unchanged even after the interaction with the optical fields. It is the measurement process that leads to the localization of the atoms. It is important that the atoms are detected in a state selective manner after their interaction with the laser fields. If, however, the total population of the states $|2\rangle$ and $|3\rangle$ is measured, there would be no position information in the result obtained. This illustrates the role of quantum coherence and interference in the CPT state.

In the following we discuss methods for detection of the CPT state. As alluded earlier the simplest possible and sufficient technique is to measure $\rho_{22}$. Alternatively, measuring the coherence $\rho_{23}$, which is proportional to $\sin k x$, can also be used to detect the CPT state. Thus, any measurement technique monitoring the coherence $\rho_{23}$ would observe a sharp decrease in the coherence if the atom passes through the nodes of the standing-wave field, leading to localization of the atom. At all other positions the coherence $\rho_{23}$ carries a non-zero value. However, ascertaining a zero of a function experimentally could be challenging as opposed to observing peaks. Therefore, a more appropriate method to measure coherence would be based on the Ramsey fringe technique. We, however, do not delve into the details of this technique.

Monitoring the population, $\rho_{22}$, of the state $|2\rangle$ can be accomplished by various techniques; we describe one in detail in the following discussion. It is based on the fluorescence shelving techniques described in [19]. It involves applying a strong drive field (see figure 3) to selectively couple the to-be-measured state to a short-lived excited state. Then monitoring the fluorescence from the excited state gives information about the population of the state to be measured.

\section{Momentum distribution of the localized atoms}

In a real experiment, as in [6], the momentum distribution of atoms would be measured. To predict the experimental results expected from our model we plot the momentum distribution, along the $x$ axis, obtained by evaluating

$$
\mathscr{P}_{2}(p)=\left|\left\langle p, 2 \mid \Psi_{\mathrm{COM}}\right\rangle\right|^{2}=\left|\int_{-\pi}^{\pi} \sqrt{\rho_{22}(x)} \mathrm{e}^{\mathrm{i} p x} \mathrm{~d} x\right|^{2}
$$

in figure 4. Here we have assumed that $f(x)=1$, i.e. uniform distribution of the atoms along the standing-wave field. Moreover, the integration is confined to a single wavelength of the standing wave. These assumptions do not cause loss of generality and the conclusions arrived at are true in general. The plots show the momentum distribution of the atoms after they are detected in the state $|2\rangle$ after interacting with the probe and a single wavelength of 


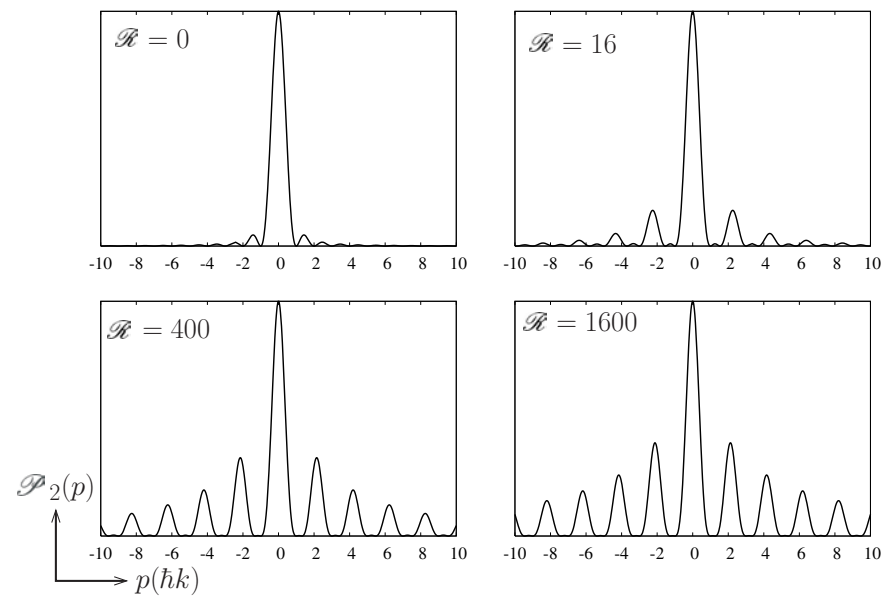

Figure 4. Momentum distribution $\mathscr{P}_{2}(p)$ in the far zone, for atoms in the state $|2\rangle$, for different values of the parameter $\mathscr{R}$.

the standing-wave field. For sharper localization, resulting due to increased $\mathscr{R}$, the higher momentum components start emerging in accordance with the Heisenberg uncertainty relation. The peaks for the plot corresponding to no localization are large in number compared to other plots, despite the amplitude being negligible for high $p$. As the localization peaks start appearing, alternate peaks in the momentum distribution start diminishing (see for example plots for $\mathscr{R}=0$ and $\mathscr{R}=16$ ). Thus, observing this feature in the momentum distribution is a clear signature of the localization of the atom. As the finesse parameter is increased higher momentum components start becoming prominent. Therefore, to remain within the Raman-Nath approximation, the highest momentum order $p \approx 10$ observed for $\mathscr{R}=1600$ should satisfy $p^{2} \hbar k_{a}^{2} / 2 m \ll \Omega_{p}$. For optical transitions of alkali atoms, $\hbar k_{a}^{2} / 2 m$ is in the $\mathrm{kHz}$ range whereas $\Omega_{p}$ is in the MHz range (table 1 in [13]), giving $p=10$ to be well within the Raman-Nath approximation.

To understand the limitations of working in the Raman-Nath regime further, we briefly discuss the case where the atomic beam has non-negligible $x$-velocity before entering the interaction region. Within the RNA regime this $x$-velocity would not change very much; nevertheless, it will limit the applicability of this protocol as the localization peaks would quickly smear out as the atoms move away from the interaction region. Therefore, in this case, the state selective detection process would have to be done very close to the interaction region.

It can also be noted that due to the phase encoding in the interaction region the atomic beam would undergo reshaping. This reshaping would be minimal as long as we are within the RNA and close to the interaction region. To ascertain localization we have proposed to measure the momentum distribution of the atoms far away from the interaction zone. This far-zone momentum distribution would be hardly affected by the beam reshaping.

\section{Applications and unique advantages}

We suggest some applications and advantages of our scheme: it can be noted that ac-Stark shift induced potential on a two-level atom due to two detuned, and counter-propagating light beams gives rise to optical lattice for trapping neutral atoms [20]. The cold neutral atoms 
in their ground states are trapped at the nodes of the standing-wave fields arising due to the counter-propagating beams. This optical-lattice trapping potential, which is proportional to $\sin ^{2}(k x)$, where $k$ is the wavenumber, is much broader than the localization feature attainable through (4). Thus, by applying our localization scheme in two dimensions ${ }^{6}$, deeper and much narrower lattice structures can be obtained. It is, however, imperative to point out that we are only describing a method to generate tightly confining potential for the optical lattice. Detailed study of the transition of the BEC to such a potential would require considerations beyond the Raman-Nath regime of the present study. Furthermore, the deep optical lattices would have to be created on a time scale much faster than the time scale over which the centre-of-mass motion of the BEC cloud evolves. Such experiments could be easily done with degenerate Bose or Fermi gases to study several predictions of the Bloch theory of solids and observation of Mott transitions [21]. It should however has to be borne in mind that state selective detection of atoms from the ensemble of atoms in a BEC would be required. Whenever this kind of detection becomes available experimentally our scheme can be readily employed to obtain new kind of optical lattices and atomic Mott insulators.

Noting the spread of the momentum distribution with stricter localization of the atom, any practical implementation of this scheme would require working in the spatial domain such that the atoms are not significantly diffracted. For example, for lithographic applications, the state selective detection and deposition of the atoms on the substrate would have to be done very close to the interaction region. This may make it necessary to use fibres to collect the fluorescence signal. This, nevertheless, does not pose any serious limitation as other state selective deposition techniques could be employed instead. The standing-wave field can be thought of as a mask for the atoms as opposed to a physical entity used in current lithographic setups. One can also envision two-dimensional (see footnote 6) spatial dependence of the light field giving rise to arbitrary two-dimensional localization pattern of atoms for lithographic applications.

Furthermore, the feature size given by our localization scheme (see equation (4)) can always be chosen to be much smaller than the width of the centre-of-mass position distribution of the entering atoms, even if the latter is considerably smaller than the wavelength of the standing light field. This provides a strong advantage for atom localization because, through monitoring of atomic population or coherence of the trapping state, we can control atomic distributions which are much smaller than the wavelength of the light fields and produce strong localization.

\section{Conclusions}

To summarize, we have devised a scheme for extreme localization of an atom as it passes through a standing-wave field, based on the phenomena of coherent population trapping. The resolution of localization peaks can be arbitrarily increased by changing the relative intensity of the probe and standing-wave control fields. The atomic population of one of the ground states forming the trapping state resembles the transfer function of Fabry-Perot cavity with controllable finesse. Increasing the finesse leads to increasing resolution for atom localization. We have discussed several methods to probe the resulting CPT state of the internal atomic states, either through selective monitoring of the population of one of the states or through the measurement of coherence. We have also pointed out the signature of the localization observable in the momentum distribution of the atoms in the far zone. It is also clarified how

6 Extension to higher dimensions could be achieved using a pair of counter-propagating laser beams for each spatial axis as in the atom trapping schemes. 
the resulting momentum distribution also helps validate the Raman-Nath approximation. We have also considered recoil due to the spontaneous emission from the excited states to validate the existence of the CPT state at the steady state. We have suggested various techniques for implementation of the model for fundamental as well as practical applications.

\section{Acknowledgments}

Part of this work, done by KTK, was carried out at the Jet Propulsion Laboratory under a contract with the National Aeronautics and Space Administration (NASA). KTK acknowledges support from the National Research Council and NASA, Codes Y and S. Also, GSA thanks E Arimondo and $G$ Rempe for useful discussions and the National Science Foundation, grant no NSF-CCF 0524673, for financial support.

\section{Appendix. The recoil effect due to spontaneous emission and existence of a pure state solution}

Rewriting the Hamiltonian (see equation (2)) of our system, where an atom is traversing through a spatially dependent standing-wave field and a uniform probe field, we have

$$
\mathscr{H}=-\hbar\left(\Omega_{p}|3\rangle\left\langle 1\left|+\Omega_{s}(x)\right| 2\right\rangle|1\rangle\right) \mathrm{e}^{-\mathrm{i} \Delta t}+\text { h.c. }
$$

The corresponding density matrix equations for the atom with the recoil effects taken into account can be written as [22]

$$
\dot{\rho}=\frac{\mathrm{i}}{\hbar}[\mathscr{H}, \rho]-\sum_{i=2,3} \frac{\gamma_{1 i}}{2}\left(|1\rangle\left\langle 1\left|\rho-2 \bar{\rho}_{11}\right| i\right\rangle\langle i|+\rho| 1\rangle\langle 1|\right)
$$

with $\gamma_{12}=\gamma_{s}$ and $\gamma_{13}=\gamma_{p}$. It can be noted that here the central term in the decay dynamics is modified, $\bar{\rho}_{11}$, as opposed to the usual, $\rho_{11}$, and it is defined through

$$
\bar{\rho}=\frac{1}{2} \int_{-1}^{+1} f(s) \mathrm{e}^{\mathrm{i} k x s} \rho \mathrm{e}^{-\mathrm{i} k x s} \mathrm{~d} s
$$

where $f(s)$ is the angular distribution of spontaneous emission and $s$ is a generalized angular coordinate. This term essentially takes into account the fact that once the atom returns to its ground state after spontaneous emission from the excited state it receives a momentum kick depending on the properties of the emitted photon.

We choose to work in the position representation, in which the above equation (A.3) describing the effect of recoil due to spontaneous emission becomes

$$
\left\langle x|\bar{\rho}| x^{\prime}\right\rangle=\int_{-1}^{+1} f(s) \mathrm{e}^{\mathrm{i} k x s} \rho\left(x, x^{\prime}\right) \mathrm{e}^{-\mathrm{i} k x s} \mathrm{~d} s \approx \mathrm{e}^{-\sigma^{2}\left(x-x^{\prime}\right)^{2}} \rho\left(x, x^{\prime}\right) .
$$

Here the approximate representation on the right-hand side is assumed for convenience and does not lead to any loss of generality of the forthcoming discussion. Here $\sigma$ is the characteristic width of the angular profile of the spontaneous emission.

To expand the density matrix equations for the internal states in the position representation we use the state labels $|i, x\rangle$ and $\left|i, x^{\prime}\right\rangle$ where $i=\{1,2,3\}$. This gives $\rho_{i, j}\left(x, x^{\prime}\right)=$ $\left\langle i, x|\rho| j, x^{\prime}\right\rangle$. Thus, the density matrix equation for all the internal degrees of freedom can be obtained by evaluating $\left\langle i, x|\dot{\rho}| j, x^{\prime}\right\rangle$. We also transform the equations to a rotating 
frame simultaneously so as to eliminate the $\mathrm{e}^{-\mathrm{i} \Delta t}$ factors. Thus, we obtain

$$
\begin{aligned}
& \dot{\rho}_{11}\left(x, x^{\prime}\right)=-\left(\gamma_{s}+\gamma_{p}\right) \rho_{11}\left(x, x^{\prime}\right)+\mathrm{i} \Omega_{p}(x) \tilde{\rho}_{13}\left(x, x^{\prime}\right)-\mathrm{i} \Omega_{p}\left(x^{\prime}\right) \tilde{\rho}_{31}\left(x, x^{\prime}\right) \\
&+\mathrm{i} \Omega_{s}(x) \tilde{\rho}_{12}\left(x, x^{\prime}\right)-\mathrm{i} \Omega_{s}\left(x^{\prime}\right) \tilde{\rho}_{21}\left(x, x^{\prime}\right), \\
& \dot{\rho}_{22}\left(x, x^{\prime}\right)= \gamma_{s}\left(x, x^{\prime}\right) \rho_{11}\left(x, x^{\prime}\right)-\gamma_{23} \rho_{22}\left(x, x^{\prime}\right)-\mathrm{i} \Omega_{s}(x) \tilde{\rho}_{12}\left(x, x^{\prime}\right)+\mathrm{i} \Omega_{s}\left(x^{\prime}\right) \tilde{\rho}_{21}\left(x, x^{\prime}\right), \\
& \dot{\rho}_{33}\left(x, x^{\prime}\right)= \gamma_{23} \rho_{22}\left(x, x^{\prime}\right)+\gamma_{p}\left(x, x^{\prime}\right) \rho_{11}\left(x, x^{\prime}\right)-\mathrm{i} \Omega_{p}(x) \tilde{\rho}_{13}\left(x, x^{\prime}\right)+\mathrm{i} \Omega_{p}\left(x^{\prime}\right) \tilde{\rho}_{31}\left(x, x^{\prime}\right), \\
& \dot{\tilde{\rho}}_{12}\left(x, x^{\prime}\right)=-\mathrm{i} \Delta \tilde{\rho}_{12}\left(x, x^{\prime}\right)-\frac{\left(\gamma_{s}+\gamma_{p}+\gamma_{23}\right)}{2} \tilde{\rho}_{12}\left(x, x^{\prime}\right)+\mathrm{i} \Omega_{p}(x) \tilde{\rho}_{32}\left(x, x^{\prime}\right) \\
& \quad+\mathrm{i} \Omega_{s}(x) \rho_{22}\left(x, x^{\prime}\right)-\mathrm{i} \Omega_{s}\left(x^{\prime}\right) \rho_{11}\left(x, x^{\prime}\right), \\
& \dot{\tilde{\rho}}_{21}\left(x, x^{\prime}\right)=\mathrm{i} \Delta \tilde{\rho}_{21}\left(x, x^{\prime}\right)-\frac{\left(\gamma_{s}+\gamma_{p}+\gamma_{23}\right)}{2} \tilde{\rho}_{21}\left(x, x^{\prime}\right)-\mathrm{i} \Omega_{p}\left(x^{\prime}\right) \tilde{\rho}_{23}\left(x, x^{\prime}\right) \quad+\mathrm{i} \Omega_{s}(x) \rho_{11}\left(x, x^{\prime}\right)-\mathrm{i} \Omega_{s}\left(x^{\prime}\right) \rho_{22}\left(x, x^{\prime}\right), \\
& \dot{\tilde{\rho}}_{13}\left(x, x^{\prime}\right)=--\mathrm{i} \Delta \tilde{\rho}_{13}\left(x, x^{\prime}\right)-\frac{\left(\gamma_{s}+\gamma_{p}\right)}{2} \tilde{\rho}_{13}\left(x, x^{\prime}\right)+\mathrm{i} \Omega_{p}(x) \rho_{33}\left(x, x^{\prime}\right) \\
& \quad \quad \mathrm{i} \Omega_{p}\left(x^{\prime}\right) \rho_{11}\left(x, x^{\prime}\right)+\mathrm{i} \Omega_{s}(x) \tilde{\rho}_{23}\left(x, x^{\prime}\right), \\
& \dot{\tilde{\rho}}_{31}\left(x, x^{\prime}\right)=\mathrm{i} \Delta \tilde{\rho}_{31}\left(x, x^{\prime}\right)-\frac{\left(\gamma_{s}+\gamma_{p}\right)}{2} \tilde{\rho}_{31}\left(x, x^{\prime}\right)+\mathrm{i} \Omega_{p}(x) \rho_{11}\left(x, x^{\prime}\right) \quad-\mathrm{i} \Omega_{p}\left(x^{\prime}\right) \rho_{33}\left(x, x^{\prime}\right)-\mathrm{i} \Omega_{s}\left(x^{\prime}\right) \tilde{\rho}_{32}\left(x, x^{\prime}\right), \\
& \dot{\tilde{\rho}}_{23}\left(x, x^{\prime}\right)=\frac{\gamma_{23}}{2} \tilde{\rho}_{23}\left(x, x^{\prime}\right)-\mathrm{i} \Omega_{p}\left(x^{\prime}\right) \tilde{\rho}_{21}\left(x, x^{\prime}\right)+\mathrm{i} \Omega_{s}(x) \tilde{\rho}_{13}\left(x, x^{\prime}\right), \\
& \dot{\tilde{\rho}}_{32}\left(x, x^{\prime}\right)=\frac{\gamma_{23}}{2} \tilde{\rho}_{32}\left(x, x^{\prime}\right)+\mathrm{i} \Omega_{p}(x) \tilde{\rho}_{12}\left(x, x^{\prime}\right)-\mathrm{i} \Omega_{s}\left(x^{\prime}\right) \tilde{\rho}_{31}\left(x, x^{\prime}\right) .
\end{aligned}
$$

Here the recoil inducing spontaneous decay events are described through

$$
\gamma_{s}\left(x, x^{\prime}\right)=\gamma_{s} \mathrm{e}^{-\sigma^{2}\left(x-x^{\prime}\right)^{2}} \quad \text { and } \quad \gamma_{p}\left(x, x^{\prime}\right)=\gamma_{p} \mathrm{e}^{-\sigma^{2}\left(x-x^{\prime}\right)^{2}} .
$$

We here use the fact that $\Omega_{p}$ is independent of $x$, and the assumption that $\gamma_{23}=0$, to solve the above equations at steady state to arrive at

$$
\rho\left(x, x^{\prime}\right)=\frac{1}{\Omega_{p}^{2}+\Omega_{s}(x) \Omega_{s}\left(x^{\prime}\right)}\left(\begin{array}{ccc}
0 & 0 & 0 \\
0 & \Omega_{p}^{2} & -\Omega_{s}\left(x^{\prime}\right) \Omega_{p} \\
0 & -\Omega_{s}(x) \Omega_{p} & \Omega_{s}(x) \Omega_{s}\left(x^{\prime}\right)
\end{array}\right)
$$

in the basis $\{|1\rangle,|2\rangle,|3\rangle\}$. Noting that $\rho\left(x, x^{\prime}\right)=|\Psi(x)\rangle\left\langle\Psi\left(x^{\prime}\right)\right|$, this density matrix can be readily identified with the state in (3). Thus, a pure state solution is obtained, even in the presence of spontaneous decay and the recoil due to it, once the system reaches the steady state.

\section{References}

[1] Stokes K D et al 1991 Phys. Rev. Lett. 671997

[2] Paspalakis E and Knight P L 2001 Phys. Rev. A 63065802 Paspalakis E, Terzis A F and Knight P L 2005 J. Mod. Opt. 521685

[3] Storey P, Collett M and Walls D 1992 Phys. Rev. Lett. 68472 Quadt R, Collett M and Walls D F 1995 Phys. Rev. Lett. 74351

[4] Kien F L, Rempe G, Schleich W P and Zubairy M S 1997 Phys. Rev. A 562972 Qamar S, Zhu S-Y and Zubairy M S 2000 Phys. Rev. A 61063806

[5] Holland M, Marksteiner S, Marte P and Zoller P 1996 Phys. Rev. Lett. 763683

[6] Kunze S, Dieckmann K and Rempe G 1997 Phys. Rev. Lett. 782038

[7] Gardner J R, Marable M L, Welch G R and Thomas J E 1993 Phys. Rev. Lett. 703404 For a review of the work in this area till the year 1995 see Thomas J E and Wang L J 1995 Phys. Rep. 262311 
[8] Sahrai M, Tajali H, Kapale K T and Zubairy M S 2005 Phys. Rev. A 72013820

[9] Kapale K T, Qamar S and Zubairy M S 2003 Phys. Rev. A 67023805

[10] Rau A V, Dunningham J A and Burnett K 2003 Science 3201081

[11] Johnson K S et al 1998 Science 2801583

[12] Boto A N et al 2000 Phys. Rev. Lett. 852733

[13] Adams C S, Sigel M and Mlynek J 1994 Phys. Rep. 240143

[14] Stenholm S 1986 Rev. Mod. Phys. 58699

[15] Phillips W D 1998 Rev. Mod. Phys. 70721

[16] Arimondo E 1996 Progress in Optics vol 35 ed E Wolf (Amsterdam: Elsevier) pp 257-354

[17] Meystre P and Sargent M III 1999 Elements of Quantum Optics 3rd edn (Berlin: Springer)

[18] Born M and Wolf E 1999 Principles of Optics: Electromagnetic Theory of Propagation, Interference, and Diffraction of Light 7th edn (Cambridge: Cambridge University Press)

[19] Blatt R and Zoller P 1988 Eur. J. Phys. 9250

[20] Jessen P S and Deutsch I H 1996 Adv. At. Mol. Opt. Phys. 3795

[21] Greiner M et al 2002 Nature 41539

[22] Dalibard J and Cohen-Tannoudji C 1985 J. Phys. B: At. Mol. Phys. 181661 